12

\title{
Осесимметричный энергоанализатор на основе электростатического декапольно-цилиндрического поля
}

\author{
(C) Ж.Т. Камбарова, ${ }^{1}$ А.А. Трубицын, ${ }^{2}$ А.О. Саулебеков ${ }^{3}$ \\ ${ }^{1}$ Карагандинский государственный университет им. акад. Е.А. Букетова, \\ 100028 Караганда, Казахстан \\ 2 Рязанский государственный радиотехнический университет, \\ 390005 Рязань, Россия \\ ${ }^{3}$ Казахстанский филиал Московского государственного университета им. М.В. Ломоносова, \\ 010010 Астана, Казахстан \\ e-mail: kambarova@bk.ru
}

(Поступило в Редакцию 13 января 2018 г.)

\begin{abstract}
Представлена новая электронно-оптическая схема осесимметричного зеркального энергоанализатора на основе электростатического декапольно-цилиндрического поля. Выполнен траекторный анализ движения электронов. Описаны конструктивные особенности схемы предложенного энергоанализатора. Численно рассчитаны режимы с фокусировкой второго порядка для двух конфигураций: 1) кольцевой источник и его изображение находятся в области внутреннего цилиндра и 2) точечный источник на оси симметрии, находящийся на большом расстоянии от энергоанализатора. Показано, что на основе декапольноцилиндрического поля может быть построен длиннофокусный зеркальный энергоанализатор с фокусировкой типа „ось-кольцо“. Для двух режимов энергоанализатора приведены аппаратные функции, оценены энергетическое разрешение и светосила устройства.
\end{abstract}

DOI: $10.21883 / J T F .2018 .11 .46636 .13-18$

\section{Введение}

Цилиндрический зеркальный анализатор энергий заряженных частиц благодаря достаточно высоким электронно-оптическим характеристикам в сочетании с простотой конструкции получил широкое применение в научных и технологических целях [1]. Одним из путей дальнейшего совершенствования этого устройства является модификация внешнего цилиндрического электрода.

Перспективным в этом направлении является серия работ Л.А. Барановой [2-5], в которых при минимальных изменениях в конструкции получено существенное улучшение фокусирующих свойств системы. В работах [2,3] предложена модифицированная конструкция цилиндрического зеркала, внешний электрод который образован отдельными электрически изолированными цилиндрами. Исследованы режимы „ось-ось“ и „кольцо-ось“ с фокусировкой пучка заряженных частиц второго порядка. Более острая фокусировка достигается в этих случаях за счет уменьшения коэффициента сферической аберрации третьего порядка по углу расходимости пучка в меридиональной плоскости на входе в анализатор.

В работе [4] предложено использовать двухкаскадный режим работы в модифицированном цилидрическом зеркале при запуске заряженных частиц через торцевую диафрагму, перпендикулярную оптической оси. Найдено, что оптимальное расстояние источника заряженных частиц от анализатора находится в пределах от 5 до $8 r_{1}$, где $r_{1}-$ радиус внутреннего цилиндра. Показано, что линейная дисперсия двухкаскадного анализатора в несколько раз превышает дисперсию однокаскадного прибора.

Усовершенствованный цилиндрический зеркальный энергоанализатор численно исследован в работе [5]. Отличие усовершенствованной конструкции от модифицированной конструкции цилиндрического зеркала, предложенной в работах [2-4], заключается в том, что как внешний, так и внутренний электроды анализатора разделены на три изолированные части каждый, вследствие чего потенциалы на отдельных частях регулируются независимо друг от друга. Данное зеркало обеспечивает большой угол захвата, равный $30^{\circ}$ при сохранении острой фокусировки в режиме фокусировки „ось-ось“, и высокую линейную дисперсию, превышающую почти в 4 раза дисперсию классического цилиндрического зеркала.

Класс потенциальных полей, названных мультипольно-цилиндрическими, впервые обоснован и классифицирован Зашкварой В.В. и Тындык Н.Н. в работах [6,7]. Мультипольно-цилиндрические поля формируются по принципу суперпозиции электростатического поля цилиндрического типа и круговых мультиполей различного порядка, нулевая эквипотенциальная поверхность которых имеет цилиндрическую форму. Применение мультипольного подхода к синтезу отклоняющих полей дает возможность для развития эффективных методов энергоанализа потоков заряженных частиц.

Ранее были достаточно детально исследованы зеркальные энергоанализаторы на основе электростатических гексапольно-цилиндрических полей, большое количество 
работ, например [8,9], в том числе монография [10], были направлены на изучение их электронно-оптических свойств и потенциальных возможностей, на выделение оптимальных схем с высоким качеством фокусировки и разрешения по энергии.

Электронно-оптическая схема электростатического зеркального декапольно-цилиндрического энергоанализатора, в котором доли цилиндрического поля и декаполя равны соответственно $\mu=1$ и $\gamma=1 / 100$, впервые описана в работе [11]. Энергоанализатор состоит из внутреннего цилиндрического электрода и внешнего отклоняющего криволинейного (выпуклого) электрода. Приближенно-аналитическим методом, описанном в [10], рассчитаны электроннооптические характеристики данного анализатора энергий заряженных частиц. Установлено, что на основе декапольно-цилиндрических полей могут быть построены светосильные энергоанализаторы с угловой фокусировкой второго порядка, если источник и его изображение находятся в области внутреннего цилиндрического электрода (тип фокусировки „кольцо-кольцо“).

В работе [12] приведены результаты численного моделирования электронно-оптической схемы электростатического декапольно-цилиндрического энергоанализатора с вкладами цилиндрического поля $\mu=1$ и кругового декаполя $\gamma=1 / 100$. Найдены два режима угловой фокусировки второго порядка типа „кольцо-кольцо“ и „ось-кольцо“. Рассчитаны фокусирующие свойства энергоанализатора.

Целями настоящей работы являются численное моделирование электронно-оптической схемы декапольноцилиндрического зеркального энергоанализатора, в котором доли цилиндрического поля и декаполя равны соответственно $\mu=3 / 2$ и $\gamma=-1 / 100$, и поиск режима фокусировки, обеспечивающего большое фокусное расстояние „образец-анализатор“.

\section{Схема декапольно-цилиндрического энергоанализатора}

Потенциал электростатического декапольно-цилиндрического поля описывается в системе координат $\rho, z$ следующим выражением:

$$
U(\rho, z)=\mu \ln (1+\rho)+\gamma U_{d},
$$

где $\ln (1+\rho)-$ потенциал цилиндрического поля, $\mu-$ коэффициент, задающий его весовой вклад,

$$
U_{d}(\rho, z)=U_{0} \rho\left(z^{4}-2 \rho^{2} z^{2}+\frac{1}{5} \rho^{4}\right)
$$

- потенциал кругового декаполя, $\gamma$ - весовой вклад кругового декаполя.

Рассчитано семейство эквипотенциальных линий электростатического декапольно-цилиндрического поля, в

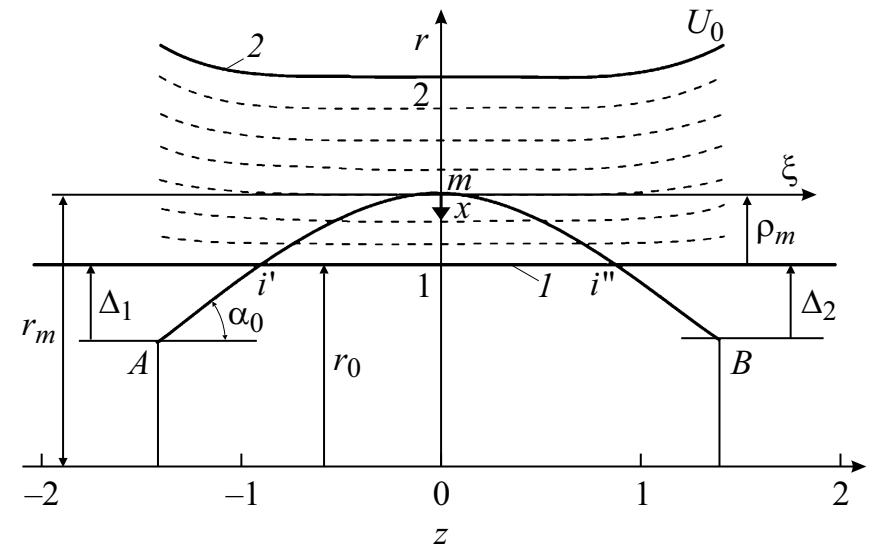

Рис. 1. Схема осесимметричного энергоанализатора на основе декапольно-цилиндрического поля при $\mu=3 / 2$ и $\gamma=-1 / 100$ : 1 - цилиндрический электрод, 2 - внешний отклоняющий электрод, $A-$ источник, $i^{\prime}-$ входная кольцевая щель, $i^{\prime \prime}-$ выходная кольцевая щель, $B-$ приемник.

котором доли цилиндрического поля и декаполя равны соответственно $\mu=3 / 2$ и $\gamma=-1 / 100$. Расчеты эквипотенциалей выполнены в среде MathCAD и приведены на рис. 1 штриховыми линиями.

Схема декапольно-цилиндрического энергоанализатора с вкладами цилиндрического поля $\mu=3 / 2$ и кругового декаполя $\gamma=-1 / 100$ представлена на рис. 1 . Вследствие малой составляющей кругового декаполя распределения эквипотенциалей декапольно-цилиндрического поля имеют небольшое отклонение от прямых линий и близки к распределению эквипотенциалей цилиндрического поля. Электростатическое декапольно- цилиндрическое поле формируется в пространстве между двумя осесимметричными коаксиальными электродами, внутренний 1 из которых имеет цилиндрическую форму (радиуса $r_{0}$ ) и находится под нулевым потенциалом, на внешний криволинейный (вогнутый) электрод 2, профиль которого повторяет эквипотенциаль декапольноцилиндрического поля, подается отклоняющий потенциал $U_{0}$.

Приближенно-аналитическим методом расчета траекторий заряженных частиц установлено, что при определенном соотношении геометрических и энергетических параметров анализатора пучок заряженных частиц, выходящий из кольцевого источника $A$, отражается полем зеркала и фокусируется в кольцевое изображение $B$.

Для дальнейших расчетов точка начала отсчета траектории переводится в ее вершину $m$ и в эту же точку размещается начало координат $x, \xi$. Все линейные размеры выдержаны в долях радиуса внутреннего цилиндрического электрода $r_{0}$

$$
\frac{r}{r_{0}}=\frac{r_{0}+r_{0} \rho}{r_{0}}=1+\rho, x=\rho_{m}-\rho, \quad \xi=\frac{z}{r_{0}} .
$$


Распределение декапольно-цилиндрического поля (1) в координатах $x, \xi$ имеет следующий вид:

$$
U_{d}(x, \xi)=U_{0} g(x, \xi),
$$

где

$$
\begin{gathered}
g(x, \xi)=\mu \ln (R-x)+\gamma\left[(R-x-1) \xi^{4}-2(R-x-1)^{3} \xi^{2}\right. \\
\left.+\frac{1}{5}(R-x-1)^{5}\right], \quad R=1+\rho_{m} .
\end{gathered}
$$

Движение заряженной частицы в поле (3) описывается системой уравнений

$$
\begin{aligned}
& m \ddot{x}=q U_{0} \varepsilon_{1}, \quad \varepsilon_{1}=-\frac{\partial g(x, \xi)}{\partial x}, \\
& m \ddot{\xi}=q U_{0} \varepsilon_{2}, \quad \varepsilon_{2}=-\frac{\partial g(x, \xi)}{\partial \xi} .
\end{aligned}
$$

Интегрируя сумму уравнений (5a) и (5b) вдоль траектории частицы в пределах от вершины $m$ до произвольной точки, придем к выражению для энергии частицы, движущейся в электростатическом поле, связывающему изменение кинетической энергии с разностью потенциалов

$$
\frac{m v_{m}^{2}}{2}-\frac{m}{2}\left(\dot{x}^{2}+\dot{\xi}^{2}\right)=-q\left(U_{m}-U(x, \xi)\right)=-q U_{0}\left(g_{0}-g_{x}\right) .
$$

Здесь $U_{m}=U_{0} g\left(x_{m}, \xi_{m}\right)=U_{0} g_{0}$ - потенциал поля в точке $m$, где $x_{m}=\xi_{m}=0, g_{x}=g(x, \xi(x))$.

Интегро-дифференциальное уравнение движения заряженной частицы в декапольно-цилиндрическом поле (3) имеет вид

$$
\left(\xi^{\prime}\right)^{2}=\frac{P^{2} \operatorname{ctg}^{2} \alpha_{0}+f_{m}-f_{x}}{g_{0}-g_{x}+f_{x}},
$$

где

$$
\begin{gathered}
g_{0}=g\left(x_{m}, \xi_{m}\right)=\mu \ln R+\frac{\gamma}{5}(R-1)^{5} \\
f_{x}=\int_{0}^{x} \frac{\partial g(x, \xi)}{\partial \xi} \xi^{\prime} d \xi=\gamma\left\{(R-x-1) \xi^{4}-2(R-x-1)^{3} \xi^{2}\right. \\
\left.+\int_{0}^{x} \xi^{4} d x-6 \int_{0}^{x}(R-x-1)^{2} \xi^{2} d x\right\}
\end{gathered}
$$

где $P_{0}^{2}=\frac{W}{q U_{0}} \sin ^{2} \alpha_{0}-$ параметр отражения, связывающий геометрические и энергетические параметры декапольно-цилиндрического зеркала.

Решение интегро-дифференциального уравнения (7) ищем в виде разложения в степенной ряд с неопределенными коэффициентами, которые рассчитываются из уравнения (7) при подстановке в него степенного ряда. Интегро-дифференциальное уравнение (7) имеет особую точку $x=0$, так как сомножитель $\left(\xi^{\prime}\right)^{2}$ в этом случае обращается в нуль, поэтому для интегрирования уравнения используется метод разложения решения уравнения $\xi$ в дробно-степенной ряд

$$
\begin{aligned}
\xi= & \sqrt{x}\left(C_{0}+C_{1} x+C_{2} x^{2}+C_{3} x^{3}+C_{4} x^{4}\right. \\
& \left.+C_{5} x^{5}+C_{6} x^{6}+\ldots\right) .
\end{aligned}
$$

Определены коэффициенты $C_{n}$, рассчитанные из условия удовлетворения уравнению (7). Радиальную составляющую точки поворота траектории $R=1+\rho_{m}$, необходимую для расчета $\xi$, можно определить, используя интегро-дифференциальное уравнение траектории (7) для точки $x=\rho_{m}$. В этом случае $\left(\xi^{\prime}\right)^{2}=\operatorname{ctg}^{2} \alpha_{0}$, $g_{x=\rho_{m}}=0$ и

$$
g_{0}+f_{m}=P^{2} .
$$

Подставив $g_{0}$ из уравнения (8) в (11), придем к выражению

$$
\mu \ln R=P^{2}-f_{m}-\frac{\gamma}{5}(R-1)^{5},
$$

из которого величина $R$ определяется методом последовательных приближений. В качестве нулевого приближения используются параметры цилиндрического зеркального анализатора

$$
R_{0}=\exp \left(P^{2}\right)=1+P^{2}+\frac{1}{2} P^{4}+\frac{1}{6} P^{6}+\frac{1}{24} P^{8}+\ldots
$$

и $f_{m_{0}}=0$.

Конечные результаты расчета траектории получены в виде разложения в ряд по величине параметра отражения зеркала $P$ с точностью до 14 порядка включительно. Bce расчеты выполнены в среде MathCAD.

Полная проекция траектории на ось симметрии $z$ от источника $A$ до его изображения $B$ есть следующая сумма:

$$
l=\frac{L}{r_{0}}=2\left(\Delta \operatorname{ctg} \alpha_{0}+\xi_{m}\right), \quad \Delta=\Delta_{1}+\Delta_{2},
$$

где $\Delta_{1}, \Delta_{2}$ - удаленность источника и его изображения от поверхности внутреннего цилиндрического электрода.

Расчет предложенного энергоанализатора приближенно-аналитическим методом также дополнен численным моделированием, которое подтвердило режим фокусировки типа „ось-кольцо“ в данной схеме.

\section{Моделирование электронно-оптической схемы декапольно-цилиндрического энергоанализатора}

Проведено численное моделирование электронно-оптической схемы декапольно-цилиндрического энергоанализатора с распределением поля $\mu=3 / 2$ и $\gamma=-1 / 100$. Численное моделирование электронно-оптической схемы декапольно-цилиндрического энергоанализатора было выполнено посредством программы „Фокус“ для 


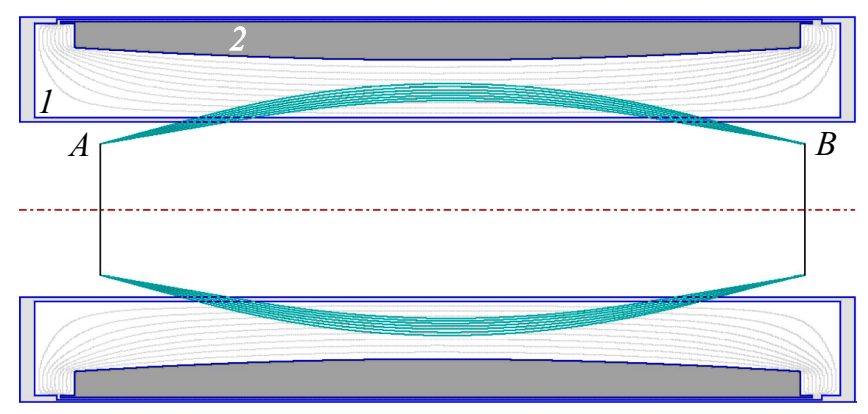

Рис. 2. Траектории электронов в схеме энергоанализатоpa с декапольно-цилиндрическим полем (режим фокусировки типа „кольцо-кольцо“): $A-$ тонкий кольцевой источник, $B$ - кольцевое изображение, 1 - цилиндрический электрод, 2 - внешний отклоняющий электрод.

автоматизированного проектирования устройств электронной и ионной оптики.

Программа „Фокус“ позволяет в графическом режиме осуществить ввод и модификацию конструкции, вычислить распределение потенциала в выделенной области и провести траекторный анализ системы [13].

На рис. 2 представлен ход траекторий электронов в схеме энергоанализатора на основе декапольноцилиндрического поля с кольцевыми источником и изображением. Длина электронно-оптической схемы составляет 11.4. Потенциал внешнего вогнутого электрода 2 равен единице. Радиус внутреннего цилиндрического электрода 1 составляет 4.2. Внутренний цилиндрический электрод под нулевым потенциалом. Отношение энергии электрона к потенциалу внешнего электрода составляет $E / U=1.3 E[\mathrm{eV}] / U[\mathrm{~V}]=1.3$. Все размеры выражены в условных единицах.

Кольцевой источник и кольцевое изображение часто необходимы по конструктивным соображениям. Кольцевой электронно-оптический источник (например, кольцевая диафрагма, облучаемая потоком частиц от образца большой площади) применяется при возбуждении электронов рентгеновским пучком в растровой ожемикроскопии и др. [14].

Согласно рис. 2, пучок электронов, выходящий из тонкого кольцевого источника $A$, отражается полем зеркала и фокусируется в кольцевое изображение $B$. Кольцевой источник $A$ и его изображение $B$ находятся в области внутреннего цилиндра. Таким образом, в схеме выполняется режим угловой фокусировки типа „кольцо-кольцо“. Из численных расчетов установлено, что в данной электронно-оптической схеме для широкого интервала изменения входных углов $\left(30-42^{\circ}\right)$ реализуется режим угловой фокусировки второго порядка с центральным углом $34.8^{\circ}$.

Для оценки энергетического разрешения энергоанализатора по результатам траекторного анализа была построена аппаратная функция. На рис. 3 представлена аппаратная функция предложенного устройства для режима угловой фокусировки типа „кольцо-кольцо“, из ана- лиза которой следует, что при светосиле $\Omega / 2 \pi=12.3 \%$ обеспечивается относительное энергетическое разрешение $R=0.8 \%$

Таким образом, данная схема с кольцевым электронно-оптическим изображением может быть использована в качестве системы, где требуется свободная от анализируемых частиц область вблизи оси, например в оже- или рентгено-электронных спектрометрах.

На рис. 4 показано множество траекторий электронов в электронно-оптической схеме декапольно-цилиндрического энергоанализатора, в котором точечный источник расположен на оси симметрии на большом расстоянии от энергоанализатора. Отношение энергии электронов к потенциалу отклоняющего электрода составляет $E / U=1.64 E[\mathrm{eV}] / U[\mathrm{~V}]=1.64$. Интервал угла ввода электронов в энергоанализатор от 32 до $40^{\circ}$. Расстояние от исследуемого образца до энергоанализатора составляет $z=3.7$.

Согласно рис. 4, вторичные электроны, эмитированные с поверхности исследуемого образца 3 под дей-

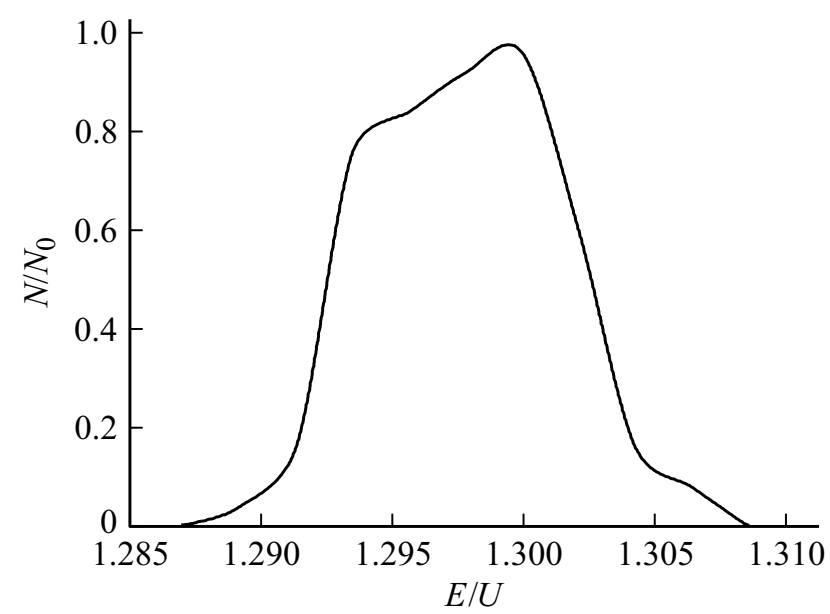

Рис. 3. Аппаратная функция энергоанализатора с декапольноцилиндрическим полем (режим угловой фокусировки типа „кольцо-кольцо“).

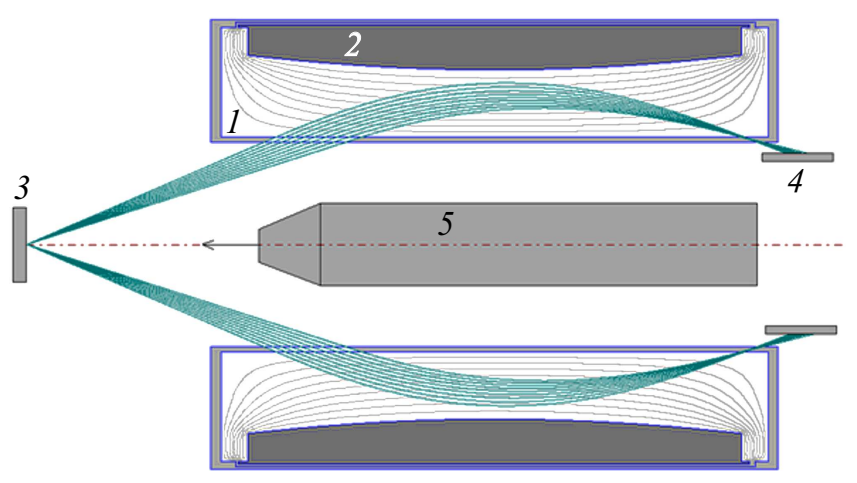

Рис. 4. Траектории электронов в схеме энергоанализатора с декапольно-цилиндрическим полем (режим фокусировки типа „ось-кольцо“): 1 - цилиндрический электрод, 2 - внешний отклоняющий электрод, 3 - исследуемый образец с точечным источником, 4 - детектор, 5 - электронная пушка. 


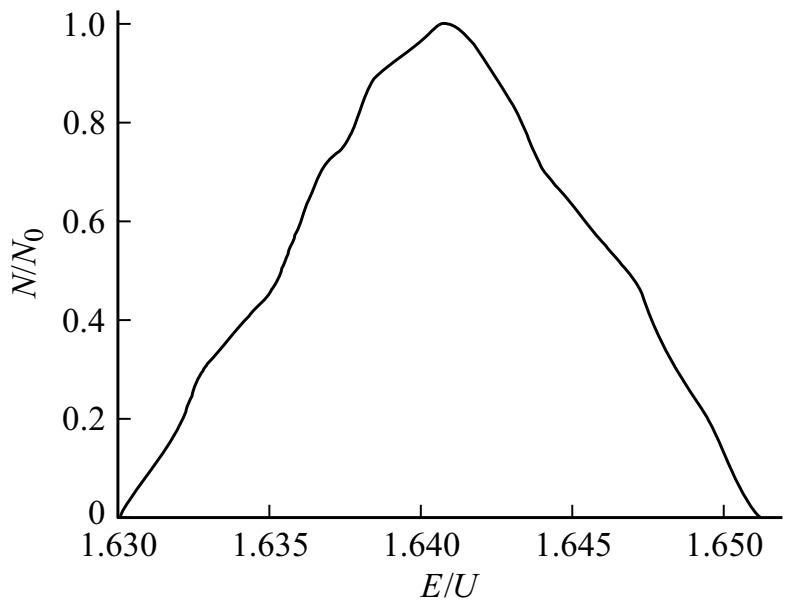

Pис. 5. Аппаратная функция энергоанализатора с декапольноцилиндрическим полем (режим угловой фокусировки типа „ось-кольцо“).

ствием первичного излучения, попадают в поле анализатора, после отражения от внешнего отклоняющего электрода 2 фокусируются в кольцевое изображение на поверхности цилиндрического электрода 1. Моделирование системы показало возможность достижения фокусировки второго порядка вблизи угла вылета из источника порядка $36.8^{\circ}$. В схеме выполняется режим угловой фокусировки второго порядка типа „ось-кольцо“.

Данная схема с режимом фокусировки типа „ось-кольцо“ обеспечивает большое фокусное расстояние „образец-анализатор“ и содержит коаксиально встроенную электронную пушку. Поскольку фокусы энергоанализатора и коаксиально встроенной в него электронной пушки при проведении экспериментов совмещаются на поверхности исследуемого образца, то пушка также предполагается длиннофокусной.

На рис. 5 показана аппаратная функция энергоанализатора с декапольно-цилиндрическим полем для режима угловой фокусировки типа „ось-кольцо“. Определено, что относительное энергетическое разрешение на полувысоте аппаратной функции устройства составляет $0.74 \%$ при светосиле $\Omega / 2 \pi=8.2 \%$.

Сравнительный анализ предложенного декапольно-цилиндрического энергоанализатора с модифицированным и усовершенствованным зеркалами (таблицы, приведенные в работах [2 и 5]) показал, что системы реализуют режим фокусировки пучка заряженных частиц второго порядка, декапольно-цилиндрический энергоанализатор уступает в разрешении, но имеет ряд других преимуществ. Преимуществом схемы энергоанализатора, реализующей режим фокусировки „ось-кольцо“, является большое фокусное расстояние „образец-анализатор“, что дает возможность пространственного совмещения, например, оже-микрозонда со средствами альтернативных методов исследований поверхности твердого тела [15]. Предложенный энергоанализатор обладает вы- сокой светосилой и разрешающей способностью, не требует дополнительной системы питания, отличается компактностью и не уступает в качестве фокусировки частиц.

Схему энергоанализатора с режимом угловой фокусировки типа „ось-кольцо“, обеспечивающую большое фокусное расстояние, можно использовать в качестве диспергирующего элемента в электронных оже-спектрометрах как основных средств элементного анализа материалов на микро- и нанометровом масштабе.

\section{Заключение}

Проведено численное моделирование схемы осесимметричного декапольно-цилиндрического энергоанализатора, в котором доли цилиндрического поля и декаполя равны соответственно $\mu=3 / 2$ и $\gamma=-1 / 100$. Получены численные модели схем энергоанализатора на основе электростатического декапольно-цилиндрического поля для двух режимов угловой фокусировки. Проведен траекторный анализ движения частиц. Построены аппаратные функции схемы для двух режимов фокусировки. Отмечено, что схема длиннофокусного энергоанализатора на основе декапольно-цилиндрического поля, дополненная встраиваемой электронной пушкой, может быть использована для локального исследования материалов и структур методом электронной оже-спектроскопии.

\section{Благодарности}

Настоящая работа была выполнена в рамках грантового финансирования по научным и научно-техническим проектам Министерства образования и науки Республики Казахстан.

\section{Список литературы}

[1] Зашквара В.В., Корсунский М.И., Космачев О.С. // ЖТФ. 1966. Т. 36. Вып. 4. С. 132-138.

[2] Баранова Л.А. // ЖТФ. 2013. Т. 83. Вып. 4. С. 149-151.

[3] Баранова Л.А. // ЖТФ. 2014. Т. 84. Вып. 3. С. 155-158.

[4] Баранова Л.А. // ЖТФ. 2016. Т. 86. Вып. 8. С. 153-155.

[5] Баранова Л.А. // ЖТФ. 2017. Т. 87. Вып. 3. С. 458-461.

[6] Зашквара В.В., Тындык Н.Н. // ЖТФ. 1991. Т. 61. Вып. 4. C. $148-157$.

[7] Zashkvara V.V., Tyndyk N.N. // Nucl. Instrum. Method. Phys. Res. 1996. A370. P. 452-460.

[8] Ashimbaeva B.U., Chokin K.Sh., Saulebekov A.O. // J. Electron. Spectroscop. Related Phenomen. 2005. Vol. 143. N 1. P. 29-32.

[9] Ashimbaeva B.U., Chokin K.Sh., Saulebekov A.O., Kambarova Zh.T. // Appl. Phys. 2012. N 2. P. 45-48.

[10] Gurov V.S., Saulebekov A.O., Trubitsyn A.A. Analytical, Approximate-Analytical, and Numerical Methods for Design of Energy Analyzers. Advances in Imaging and Electron Physics. - Academic Press is an imprint of Elsevier Toulouse, France, 2015. P. 224. 
[11] Аиимбаева Б.У., Чокин К.Ш., Саулебеков А.О., Камбарова Ж.T. // Вестник Карагандинского ун-та. Серия физика. 2012. № 4 (68). C. 86-92.

[12] Kambarova Z.T., Saulebekov A.O., Saulebekova D.A. // B сборнике: IOP Conference Series: Materials Science and Engineering 12. Cep. „XII International Conference Radiation-Thermal Effects and Processes in Inorganic Materials“ 2017. C. 012078. DOI:10.1088/1757-899X/168/1/012078

[13] Trubitsyn A., Grachev E., Gurov V., Bochkov I., Bochkov V. // Proceedings of SPIE. 2017. Vol. 10250. P. 0V-1-0V-7.

[14] Меньшиков К.А. // ЖТФ. 1982. Т. 52. Вып. 11. С. 22452252.

[15] Трубицын А.А., Суворов Д.В., Тарабрин Д.Ю., Кукса П.И., Саулебеков А.О., Камбарова Ж.Т. // Вестник РГРТУ. 2012. Вып. 42. № 4. Ч. 1. С. 54-59. 